\title{
Magnetron Sputter-Coated Nanoparticle MoS2 Supported on Nanocarbon: A Highly Efficient Electrocatalyst toward the Hydrogen Evolution Reaction
}

\author{
Samuel J. Rowley-Neale,,,$\ddagger$ Marina Ratova, $\uparrow$ Lucas T. N. Fugita,§ Graham C. Smith, // \\ Amer Gaffar,,,$\ddagger$ Justyna Kulczyk-Malecka,,,$\ddagger$ Peter J. Kelly,,,$\ddagger$ and Craig E. Banks ${ }^{\star}, \dagger, \ddagger$
}

†Faculty of Science and Engineering and „Manchester Fuel Cell Innovation Centre, Manchester Metropolitan University, Chester

Street, Manchester M1 5GD, U.K.

§University of Sa o Paulo, 580, Prof. Lineu Prestes Avenue, Butanta , Sa o Paulo 05508-000, SP, Brazil // Department of Natural Sciences, Faculty of Science and Engineering, University of Chester, Thornton Science Park, Pool Lane,

Ince, Chester CH2 4NU, U.K.

ABSTRACT: The design and fabrication of inexpensive highly efficient electrocatalysts for the production of hydrogen via the hydrogen evolution reaction (HER) underpin a plethora of emerging clean energy technologies. Herein, we report the fabrication of highly efficient electrocatalysts for the HER based on magnetron-sputtered MoS2 onto a nanocarbon support, termed MoS2/C. Magnetron sputtering time is explored as a function of its physiochemical composition and HER performance; increased sputtering times give rise to materials with differing compositions, i.e., Mo4+ to Mo6+ and associated S anions (sulfide, elemental, and sulfate), and improved HER outputs. An optimized sputtering time of $45 \mathrm{~min}$ was used to fabricate the MoS2/C material. This gave rise to an optimal HER performance with regard to its HER onset potential, achievable current, and Tafel value, which were -0.44 (vs saturated calomel electrode (SCE)), $-1.45 \mathrm{mV} \mathrm{s}-1$, and $43 \mathrm{mV}$ dec-1, respectively, which has the highest composition of Mo4+ and sulfide (MoS2). Electrochemical testing toward the HER via drop casting MoS2/C upon screen-printed electrodes (SPEs) to electrically wire the nanomaterial is found to be mass coverage dependent, where the current density increases up to a critical mass (ca. $50 \mu \mathrm{g} \mathrm{cm}-2$ ), after which a plateau is observed. To allow for a translation of the bespoke fabricated MoS2/C from laboratory to new industrial applications, MoS2/C was incorporated into the bulk ink utilized in the fabrication of SPEs (denoted as MoS2/C-SPE), thus allowing for improved electrical wiring to the MoS2/C and resulting in the production of scalable and reproducible electrocatalytic platforms. The MoS2/C-SPEs displayed far greater HER catalysis with a $450 \mathrm{mV}$ reduction in the HER onset potential and a $1.70 \mathrm{~mA} \mathrm{~cm}-2$ increase in the achievable current density (recorded at $-0.75 \mathrm{~V}$ (VS SCE)), compared to a bare/unmodified graphitic SPE. The approach of using magnetron sputtering to modify carbon with MoS2 facilitates the production of massproducible, stable, and effective electrode materials for possible use in electrolyzers, which are cost competitive to $\mathrm{Pt}$ and mitigate the need to use time-consuming and low-yield exfoliation techniques typically used to fabricate pristine MoS2.

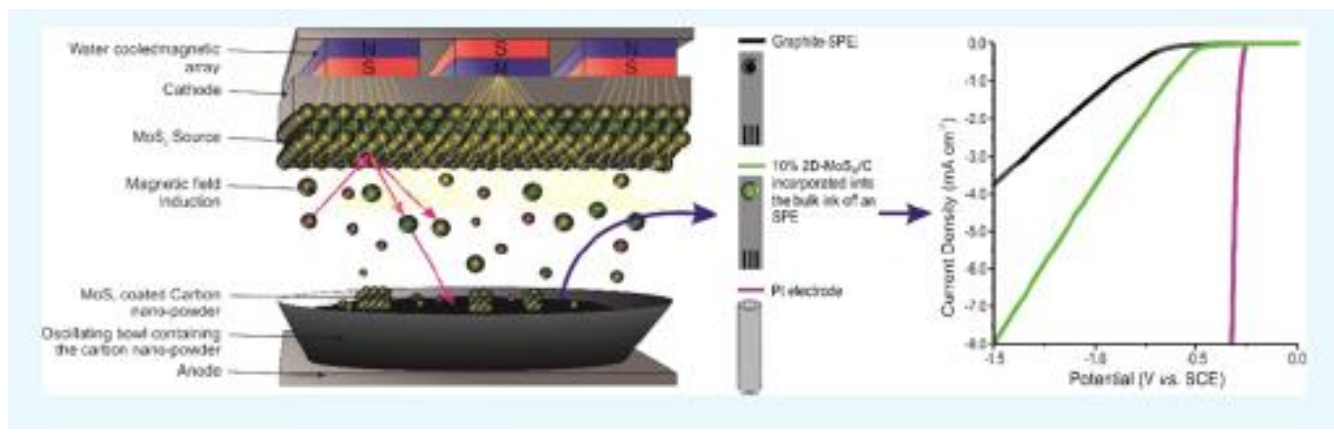




\section{INTRODUCTION}

The current fossil fuel (FF)-based energy economy has resulted in several detrimental issues, including poor air quality within heavily urbanized areas and anthropogenic climate change. As a result of this, research has focused on finding less polluting and more sustainable alternative energy generation/ storage methods. ${ }^{1}$ Although there are numerous possible alternatives, very few are economically competitive with their FF counterparts. A promising candidate that could become cost competitive with FF is hydrogen, 2 produced via the hydrogen evolution reaction (HER) $\left(2 \mathrm{H}^{+}+2 \mathrm{e}-\rightarrow\right.$ $\mathrm{H}_{2}$ ) in electrolyzers, which can then be used as a fuel source in fuel cells. The requirement of expensive platinum $(\mathrm{Pt})$ as an effective electrocatalyst within electrolyzers is becoming nonessential as researchers have shown that two-dimensional (2D) nanomaterials, such as $\mathrm{MoS}_{2}$ and $\mathrm{MoSe}_{2}$, for example, can offer comparable activity, in regard to the HER onset potential and achievable current densities, while being cheaper and more earth abundant. ${ }^{3-5}$ For example, Voiry et al. ${ }^{6}$ showed that conductive 1-T MoS 2 nanosheets can display HER activity compared with Pt-based electrodes. A recent study by Lazar and Otyepka ${ }^{7}$ has added compelling evidence to the theory that MoS2 is anisotropic in regard to its electrochemical properties, with the edge planes being the site of electron transfer and the basal planes being considered comparatively inert due to their negligible contribution to the heterogeneous electron transfer kinetics displayed by the $\mathrm{MoS}_{2}$ monolayer. The edge plane is typically composed of Mo and $\mathrm{S}$ atoms, both have unique electrocatalytic properties under particular conditions. ${ }^{7-10}$ In the case of the HER in an acidic medium, it is the dangling bonds of the electronegatively charged $\mathrm{S}$ atoms, located at the monolayers edge sites, which have an affinity for binding electropositive $\mathrm{H}^{+}$atoms. This affinity for $\mathrm{H}+$ adsorption is due to low binding energy $(+0.08 \mathrm{eV})$, predicted by density functional theory, at the edge plane sites. ${ }^{8,11,12}$

There are multiple fabrication techniques utilized within the literature for the production of twodimensional dichalcogenides, such as MoS2 nanosheets, the most common fabrication techniques being chemical vapor deposition, ${ }^{13}$ liquid exfoliation, ${ }^{14}$ mechanical exfoliation, ${ }^{15}$ electrochemical exfoliation, and shear exfoliation. ${ }^{16}$ One interesting approach is the use of magnetron sputtering, which is a well-established technique for the deposition of thin films. The use of magnetron sputtering enables precise control over the amount and composition of the sputtered material, whereas variation of the deposition parameters, such as power, pulse frequency, and deposition pressure, allows control of the coating morphological and structural properties. ${ }^{17}$ Magnetron sputtering is typically used for deposition of thin films onto flat substrates, but recent work described by Ratova et al. ${ }^{18,19}$ enables simple, yet efficient deposition of sputtered coatings onto powders or particulates. Related to the context of this work, Escalera-López et al. ${ }^{20}$ reported the fabrication of a Ni-MoS hybrid of nanoclusters supported upon a glassy carbon stub using a dualtarget magnetron sputtering technique and explored these nanoclusters toward the HER. The $\mathrm{Ni}-\mathrm{MoS}_{2}$ nanoclusters were shown to display a $100 \mathrm{mV}$ reduction in the HER onset potential and a 3fold increase in the exchange current density compared to undoped $\mathrm{MoS}_{2}$ clusters. In this paper, we report for the first time, the fabrication of novel and highly efficient electrocatalysts for the HER via magnetron sputtering of MoS2 onto nanocarbon supports $\left(\mathrm{MoS}_{2} / \mathrm{C}\right)$. The composition of the $\mathrm{MoS}_{2} / \mathrm{C}$ is detailed as a function of sputtering time (7.5-120 $\mathrm{min})$, and its effect upon the HER is evaluated. The optimal MoS2/C sputtering is evaluated toward the HER and is explored as a function of coverage upon screen-printed electrodes (SPEs). Additionally, $\mathrm{MoS}_{2} / \mathrm{C}$ is incorporated into the bulk ink utilized in the fabrication of bespoke SPEs, allowing for the mass production of reproducible electrocatalytic platforms to be realized for the first time.

\section{RESULTS AND DISCUSSION}

The $\mathrm{MoS}_{2} / \mathrm{C}$ was fabricated, as described in the Experimental Section, via magnetron sputtering. $\mathrm{MoS}_{2}$ was deposited onto the nanocarbon support for different sputtering times over the range of 7.5-120 min. The resultant bespoke material was explored electrochemically toward the HER within 
an acidic media via drop casting a dispersal onto screen-printed electrodes (SPEs) to electrochemically wire and study the MoS2/C. Figure 1 depicts typical linear sweep voltammograms (LSVs) corresponding to the HER as a function of different sputtering times, where it is readily evident that the bare/ unmodified SPE has a more electronegative onset potential (-0.84 V (vs saturated calomel electrode (SCE))) than any of the MoS2/C variants, which are closer to the optimal polycrystalline platinum ( $\mathrm{Pt}$ ) electrode that exhibits an electrochemical signature at $-0.28 \mathrm{~V}$. In regard to the MoS2/C electrochemical activity, from 7.5 to $45 \mathrm{~min}$ of deposition, the HER onset overpotential decreases in electronegativity from -0.80 to $-0.44 \mathrm{~V}$ (VS SCE). For deposition times greater than $45 \mathrm{~min}$, there is a gradual increase in the electronegativity of the overpotential for the HER to $-0.46 \mathrm{~V}$ (vs SCE) and $-0.50 \mathrm{~V}$ (vs SCE) for the 60 and $120 \mathrm{~min}$ MoS2/C variants, respectively. It was also important to consider which deposition time resulted in a MoS2/C variant, that once deposited onto a SPEs surface, resulted in the greatest achievable current density. As presented in Table 1, the current density follows a similar trend to the HER onset potential, with the current density recorded at $-0.75 \mathrm{~V}$ (VS SCE), increasing from -0.11 to $-1.45 \mathrm{~mA} \mathrm{~cm}-2$ as the deposition time increases from 7.5 to $45 \mathrm{~min}$, respectively. Again, with deposition times over $45 \mathrm{~min}$, there was a decrease in HER activity as the 60 and 120 min runs exhibited achievable current densities of -1.30 and $-1.19 \mathrm{~mA} \mathrm{~cm}-2$, respectively. With the clear increase in the HER activity of the SPEs upon modification with $\mathrm{MoS}_{2} / \mathrm{C}$ variants, especially the $45 \mathrm{~min}$ variant, it is important to assess whether there is an alteration in the HER mechanism between the bare/ unmodified SPE and the $\mathrm{MoS}_{2} / \mathrm{C}$ variants. 


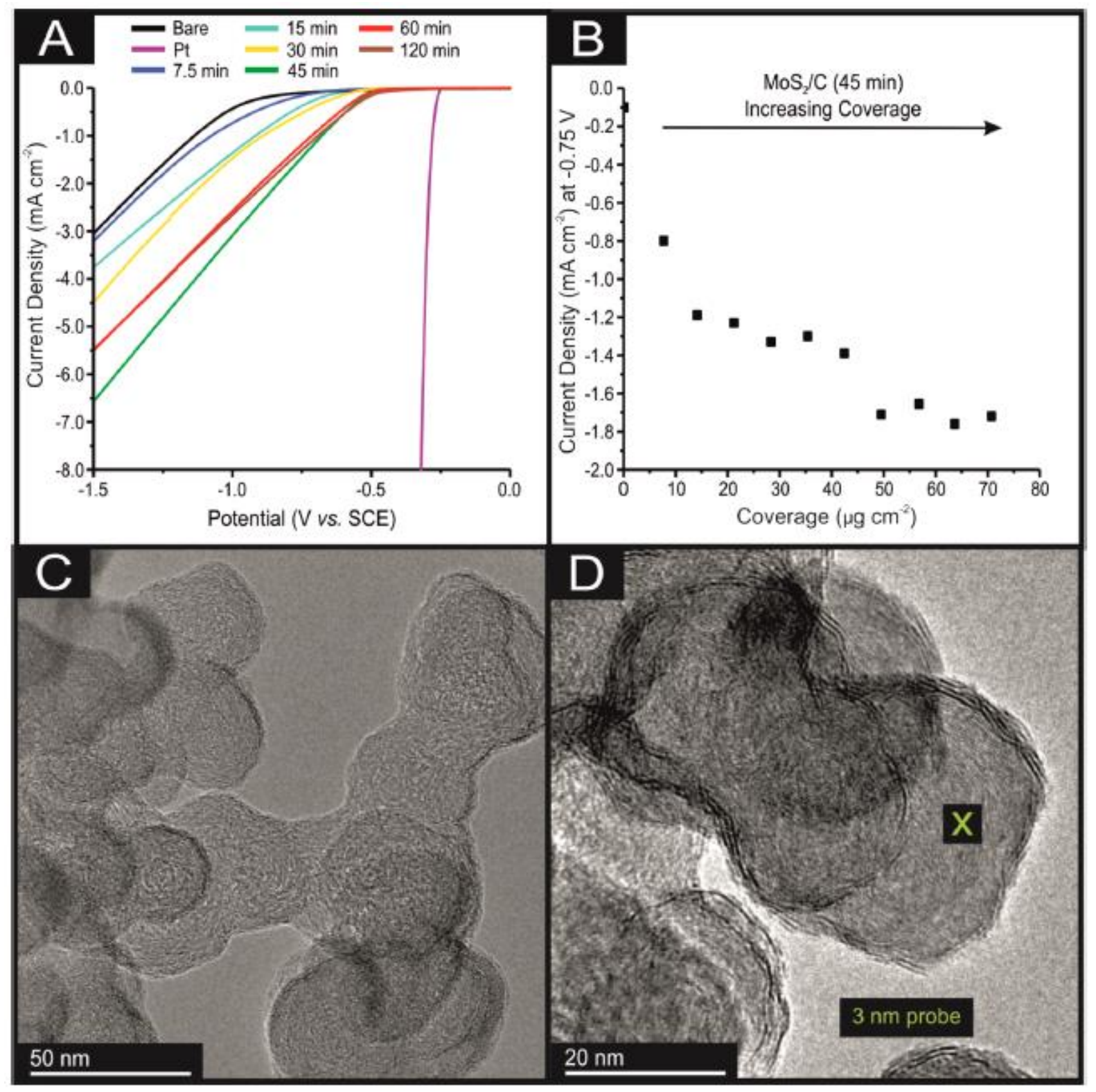

Figure 1. (A) Linear sweep voltammograms (LSVs) showing the HER activity of a bare/unmodified SPE, polycrystalline platinum electrode, and SPEs that have been modified (via drop casting) with 14 $\mu \mathrm{g} \mathrm{cm}-2$ of the carbon nanopowder (no $\mathrm{MoS}_{2}$ ) and $\mathrm{MoS}_{2} / \mathrm{C}$, which has been magnetronsputtered for $7.5,15,30,45,60$, and 120 min. For comparative purposes, a bare/unmodified SPE and a polycrystalline Pt electrode were also tested. Solution composition: $0.5 \mathrm{M} \mathrm{H} 2 \mathrm{SO} 4$; scan rate: $25 \mathrm{mV}$ s-1 (vs SCE). (B) Coverage study of 0, 7.1, 14.1, 21.2, 28.3, 35.4, 49.5, 56.8, 63.6, and $70.7 \mu \mathrm{g} \mathrm{cm}-2$ of the optimized $45 \mathrm{~min}$ MoS2/C variant electrically wired via drop casting onto SPEs. (C, D) Transmission electron microscopy (TEM) of the nanocarbon after magnetron sputter deposition of MoS2 for $45 \mathrm{~min}$. Example of an energy-dispersive X-ray (EDX) sample spot is shown in (D).

To explore the HER mechanism occurring at the MoS2/C modified SPEs, Tafel analysis was performed as this is a common approach within the academic literature. ${ }^{5,21}$ There are three possible steps in the HER reaction, each of which is capable of being the rate-determining step. The initial $\mathrm{H}+$ discharge step being the Volmer reaction, which is followed by one of two possible discharge steps, either the Heyrovsky or the Tafel step. ${ }^{22}$ An optimal HER electrocatalyst, such as Pt, is expected to have a discharge step as the rate-limiting step in the HER mechanism, which is typically limited by 
the rate of the Tafel step. Tafel analysis was performed on the Faradaic sections of the LSVs shown in Figure $1 \mathrm{~A}$ with the resultant Tafel slopes and values being exhibited in Figure S1 and Table 1, respectively. The bare/unmodified SPE and Pt electrode were observed to have Tafel slope values of 133 and $18 \mathrm{mV}$ dec-1, respectively; thus, it is likely that the rate-limiting steps in these cases are the Volmer adsorption and Tafel discharge step, respectively. The 7.5 and $15 \mathrm{~min}$ MoS2/C variants both display Tafel slope values, which indicate that they are rate limited by the Volmer step, whereas the $30,45,60$, and $120 \mathrm{~min}$ MoS2/C variants have values indicating that they are limited by the Heyrovsky step. These values imply that, upon modification of an SPE with the 30, 45, 60, and 120 $\operatorname{min~} \mathrm{MoS}_{2} / \mathrm{C}$ variants, there is a reduction in the free energy barrier of the discharge step and the HER reaction mechanism is beneficially altered ${ }^{23}$ It is clear from the above analysis that utilizing a MoS2 deposition time of 45 min results in the $\mathrm{MoS}_{2} / \mathrm{C}$ variant with the most beneficial HER activity, with regard to it displaying the least electronegative onset potential, highest current density, and smallest Tafel slope values of $-0.44 \mathrm{~V}$ ( $\mathrm{vs} \mathrm{SCE}$ ), $-1.45 \mathrm{~mA} \mathrm{~cm}-2$, and $43 \mathrm{mV} \mathrm{dec}-1$. It is of note that these values are preferential (greater HER activity) to those obtained when an equivalent mass coverage of commercially available pristine 2D-MoS is deposited onto a SPE (LSV's not presented herein but data reported in Table 1). This is likely due to a higher surface coverage of $\mathrm{MoS}_{2}$ on the nanocarbon surface from the 45 min sputtering time, which is corroborated with X-ray photoelectron spectroscopy (XPS) analysis (see Table 2 and Figures S2 and S3). Thus, the above data indicate that the $45 \mathrm{~min} \mathrm{MoS}_{2} / \mathrm{C}$ variant has the most abundant electrocatalytic sites upon the surface of the $\mathrm{MoS}_{2} / \mathrm{C}$, where the active edges (S atoms) of the MoS2 are preferentially exposed and capable of binding protons. The reduction in HER activity after 45 min likely represents a critical deposition point, where additional deposition of $\mathrm{MoS}_{2}$ leads to blocking of the active edge sites by less-electrocatalytic Mo- and S-containing compounds (Table 1).

Table 1. Summary of the HER Performance of the Fabricated $\mathrm{MoS}_{2} / \mathrm{C}$ Materials and Comparison to the Academic Literature/Controls ${ }^{a}$

\begin{tabular}{|c|c|c|c|}
\hline $\begin{array}{l}\mathrm{MoS}_{2} \text { material on an } \\
\text { SPE }\left(14 \mu \mathrm{g} \mathrm{cm}^{-2}\right)\end{array}$ & $\begin{array}{l}\text { Tafel slope } \\
\left(\mathrm{mV} \mathrm{dec}^{-1}\right)\end{array}$ & $\begin{array}{c}\text { current density } \\
(\mathrm{mA} \mathrm{cm}-2) \text { at } \\
-0.75 \mathrm{~V}\end{array}$ & $\begin{array}{l}\text { HER onset } \\
(\mathrm{V} \text { vs SCE) }\end{array}$ \\
\hline $2 \mathrm{~h} \mathrm{MoS}_{2} / \mathrm{C}$ & 47 & -1.19 & -0.50 \\
\hline $1 \mathrm{~h} \mathrm{MoS}_{2} / \mathrm{C}$ & 50 & -1.30 & -0.46 \\
\hline $45 \mathrm{~min} \mathrm{MoS}_{2} / \mathrm{C}$ & 43 & -1.45 & -0.44 \\
\hline $30 \mathrm{~min} \mathrm{MoS}_{2} / \mathrm{C}$ & 50 & -0.53 & -0.49 \\
\hline $15 \mathrm{~min} \mathrm{MoS}_{2} / \mathrm{C}$ & 104 & -0.16 & -0.61 \\
\hline $7.5 \mathrm{~min} \mathrm{MoS}_{2} / \mathrm{C}$ & 121 & -0.11 & -0.80 \\
\hline $2.5 \% \mathrm{MoS}_{2} / \mathrm{C}-\mathrm{SPE}$ & 59 & -0.93 & -0.49 \\
\hline $5.0 \% \mathrm{MoS}_{2} / \mathrm{C}-\mathrm{SPE}$ & 53 & -1.16 & -0.48 \\
\hline $7.5 \% \mathrm{MoS}_{2} / \mathrm{C}-\mathrm{SPE}$ & 44 & -1.58 & -0.45 \\
\hline $10 \% \mathrm{MoS}_{2} / \mathrm{C}-\mathrm{SPE}$ & 43 & -1.81 & -0.45 \\
\hline $\begin{array}{l}\text { controls: pristine } \\
2 \mathrm{D}-\mathrm{MoS}_{2}{ }^{\boldsymbol{\Lambda}}\end{array}$ & 92 & -1.284 & -0.46 \\
\hline carbon nanopowder $\boldsymbol{\Lambda}$ & 125 & -0.13 & -0.82 \\
\hline bare/unmodified SPE & 133 & -0.12 & -0.84 \\
\hline polycrystalline $\mathrm{Pt}$ & 18 & $*$ & -0.28 \\
\hline
\end{tabular}

aKey: *; current density too large to be recorded on potentiostat used; SPE: screen-printed electrode; $\boldsymbol{\Lambda}$; $14 \mu \mathrm{g} \mathrm{cm}^{-2}$ mass coverage deposited via drop casting; Pt: platinum. 
Figure 1B demonstrates how the immobilized mass/coverage of $\mathrm{MoS}_{2} / \mathrm{C}$ on an SPE affects achievable current density (recorded at $-0.75 \mathrm{~V}$ (vs SCE)). There is a significant increase from -0.10 to $-0.81 \mathrm{~mA}$ $\mathrm{cm}-2$ in the achievable current density upon modification of a bare SPE with ca. $7 \mu \mathrm{g} \mathrm{cm}-2$.

Following this, there is then a gradual increase until at ca. $50 \mu \mathrm{g} \mathrm{cm}-2$ where the current density is $-1.71 \mathrm{~mA} \mathrm{~cm}-2$. With additional masses/coverage, a plateauing is observed where further increases in MoS2/C coverage do not result in any significant increase in the achievable current density. This obvious plateau likely arises when the coverage of MoS2/C is sufficient to result in an optimal ratio of edge-to-basal MoS2 sites after which further additions do not infer greater HER catalysis due to reduction in the accessible triple phase boundary. The plateauing may also arise due to delamination of MoS2/C from the SPEs surface, which has been observed in other systems/configurations. ${ }^{24}$

A thorough physicochemical characterization of the fabricated $\mathrm{MoS}_{2} / \mathrm{C}$ was performed to evaluate the observed alterations in HER activity as a function of magnetron sputtering time, i.e., nanoparticle coverage. Figure S4 shows SEM images of the 7.5, 15, 30, 45, 60, and 120 min magnetron sputtercoated $\mathrm{MoS}_{2} / \mathrm{C}$. TEM was performed on the optimized (see electrochemical characterization) $45 \mathrm{~min}$ $\mathrm{MoS}_{2} / \mathrm{C}$ sample with TEM images depicted in Figure 1C,D. Note that Fourier transforms for the 45 min $\mathrm{MoS}_{2} / \mathrm{C}$ variant can be observed in Figure S5. It is clear that the individual particles are in the range of $50 \mathrm{~nm}$, which agrees with the manufacturer's quoted size range for uncoated nanocarbon of $40-45 \mathrm{~nm}$ with magnetron sputtering coating the outside of the nanocarbons, resulting in a final size of $50 \mathrm{~nm}$ for the MoS2/C; interestingly, it can be visually observed that there is a layered nanomaterial around the circumference of the $45 \mathrm{~min}$ MoS2/C particles (see Figure 1D). The layers around the circumference of the carbon particles have an interlayer distance of ca. $0.5 \mathrm{~nm}$, which corresponds with the expected value for MoS2 nanosheets. ${ }^{25}$

Raman spectroscopy was also performed on the MoS2/C variants (spectra shown in Figure S6), where the expected peaks at ca. 380 and $405 \mathrm{~cm}^{-1}$, which correspond to the $\mathrm{E} 12 \mathrm{~g}$ and A1g, of MoS respectively, are unexpectedly absent. ${ }^{26}$ Raman peaks are observed at ca. 1350 and $1580 \mathrm{~cm}^{-1}$, which are characteristic of a graphite-based material. ${ }^{27,28}$ Additionally, X-ray diffraction (XRD) analysis was conducted but the characteristic diffraction peak for $\mathrm{MoS}_{2}$ at $14.2^{\circ}$ was not observed; ${ }^{29}$ the XRD spectra for each $\mathrm{MoS}_{2} / \mathrm{C}$ variant is presented in Figure S7. EDX was additionally performed with the average $(\mathrm{N}=5)$ percentage of carbon, molybdenum, and sulfur being shown in Table S1 (see Figure 1D for an example of an EDX analysis spot). As the deposition time for the $\mathrm{MoS}_{2}$ increases from 7.5 to $120 \mathrm{~min}$, there is a corresponding increase in the total molybdenum and sulfur concentration from ca. 0.3 to $1.71 \%$. The atomic ratio of molybdenum to sulfur at every deposition time is expected to be ca. 1:2, which strongly indicates that the magnetron sputtering technique utilized herein is in fact depositing $\operatorname{MoS}_{2} \cdot{ }^{30}$ Note that while the physiochemical characterization (Raman and XRD) fails to clearly identify the $\mathrm{MoS}_{2}$, it is clearly present as evidenced by the EDX and electrochemical analysis, noting that the latter is a very sensitive interfacial technique. Finally, XPS was performed on the $\mathrm{MoS}_{2} / \mathrm{C}$ variants. In all cases, only C, O, Mo, and S were detected with a typical survey spectrum shown in Figures $\mathrm{S} 1$ and $\mathrm{S} 2$ for the $45 \mathrm{~min}$ sputtered sample. In addition to the strong $C 1 s, O$ 1s, Mo 3d, and S $2 p$ lines, the spectrum also shows the Mo 3p doublet at ca. 394 and $412 \mathrm{eV}$, the Mo 3 s peak at ca. $506 \mathrm{eV}$, the X-ray-excited O KVV Auger peak at ca. $990 \mathrm{eV}$, and weaker structures at low binding energy attributable to Mo $4 \mathrm{~s}$ and $4 \mathrm{p}$ peaks, along with weak $\mathrm{O} 2 \mathrm{~s}$ and $\mathrm{C} 2 \mathrm{~s} / 2 \mathrm{p}$ contributions. The high-resolution spectra for the Mo and S areas of the $\mathrm{MoS}_{2} / \mathrm{C} 45 \mathrm{~min}$ sample is presented in Figure $\mathrm{S} 3$ and used as a representative example for the other deposition times. For a thorough description of the Mo and S spectra, interested readers are directed to the Supporting Information. The chemical state and elemental composition, expressed in a percentage, are determined and presented in Table 2 . 
Table 2. Elemental Composition of the $\mathrm{MoS}_{2} / \mathrm{C}$ Variants Deduced via XPS Analysis as a Function of Magnetron Sputtering Time

\begin{tabular}{lrrrrrc} 
& \multicolumn{7}{c}{ time $(\mathrm{min})$} \\
\cline { 2 - 7 } \multicolumn{1}{c}{ element } & \multicolumn{1}{c}{7.5} & \multicolumn{1}{c}{15} & \multicolumn{1}{c}{30} & \multicolumn{1}{c}{45} & 60 & 120 \\
C 1s & 91.01 & 85.07 & 86.84 & 83.32 & 91.38 & 91.39 \\
O 1s & 5.47 & 7.43 & 7.19 & 6.74 & 4.79 & 5.34 \\
Mo $^{4+}$ & 0.43 & 1.32 & 1.04 & 2.06 & 0.55 & 0.5 \\
Mo $^{6+}$ & 0.85 & 1.21 & 1.03 & 0.9 & 0.51 & 0.41 \\
S (sulfide) & 0.72 & 2.25 & 1.66 & 4.17 & 0.97 & 1.08 \\
S (elemental) & 0.81 & 1.88 & 1.29 & 1.79 & 0.68 & 0.59 \\
S (sulfate) & 0.71 & 0.84 & 0.96 & 1.02 & 1.12 & 0.69
\end{tabular}

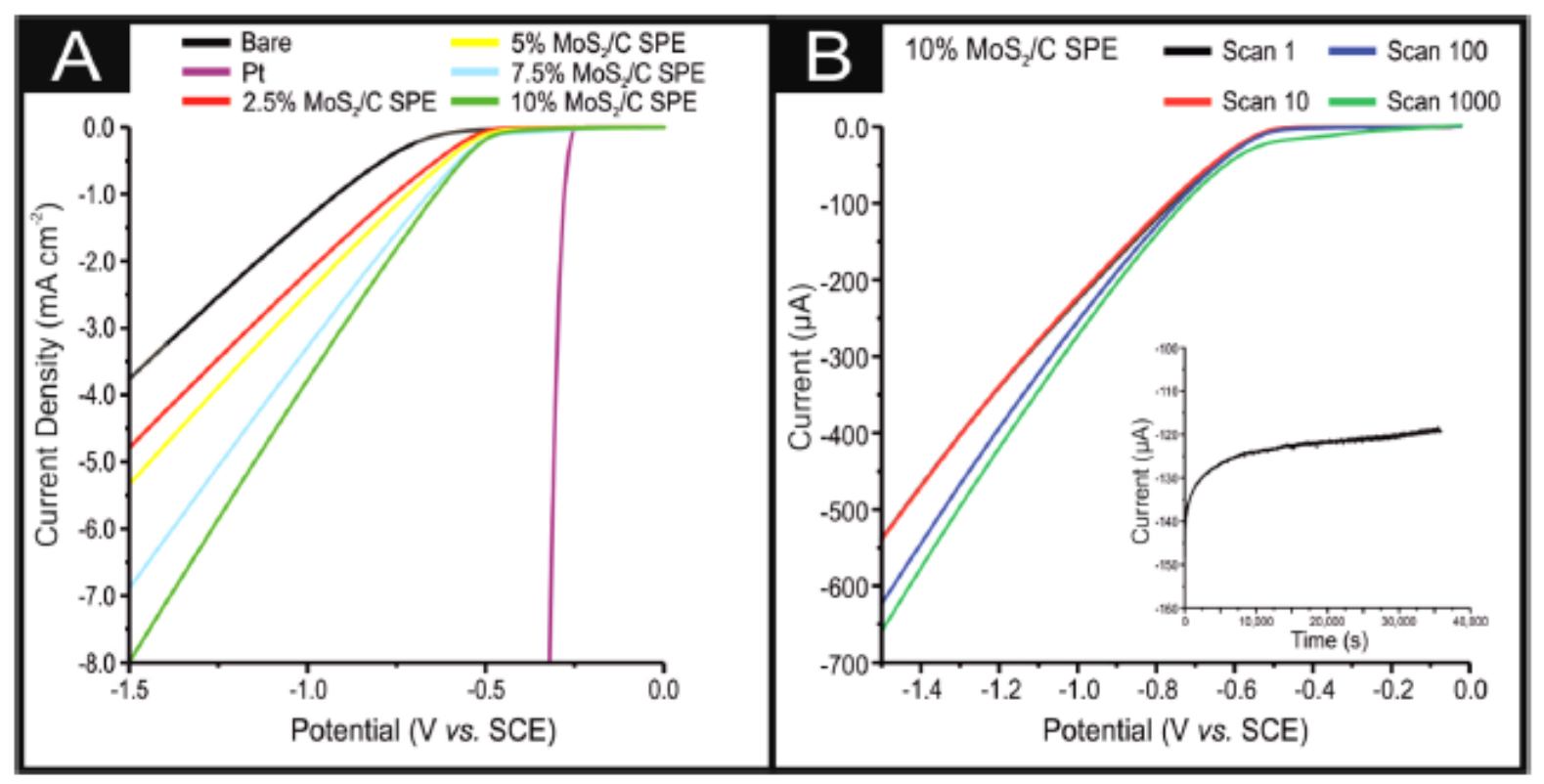

Figure 2. (A) Linear sweep voltammograms (LSVs) showing the HER activity of a bare/unmodified SPE, polycrystalline platinum electrode, and the $2.5,5.0,7.5$, and $10.0 \%$ MoS2/C-SPEs produced using the optimized $45 \mathrm{~min}$ MoS2/C. Solution composition: $0.5 \mathrm{M} \mathrm{H} 2 \mathrm{~S} 4$; scan rate: $25 \mathrm{mVs}-1$. (B) Tafel slopes corresponding to the Faradaic regions of the LSVs shown in (A). Solution composition: $0.5 \mathrm{M} \mathrm{H} 2 \mathrm{SO}$, scan rate: $25 \mathrm{mV} \mathrm{s}-1$ (vs SCE). (B) Cyclic stability examination of a 10\% MoS2/C-SPE via LSV (scan rate: $100 \mathrm{mV} \mathrm{s}-1$ (Vs SCE)) was performed between the potential range of 0 and $-1.4 \mathrm{~V}$, repeated for 1000 cycles; this figure shows the first scan (black line), 10th (red line) scan, 100th (blue) scan, and $1000^{\text {th }}$ scan (green line). Note that the inset displays the current output achieved when the potential was held at $-0.75 \mathrm{~V}$ (vs SCE).

It is not possible to determine the exact amounts of $\mathrm{MoS}_{2}, \mathrm{MoO}_{3}, \mathrm{MoSO}_{4}$, and other Mo/S molecules on the surface of the $\mathrm{MoS}_{2} / \mathrm{C}$ samples. However, balancing the oxygen and sulfur concentrations with the relative amounts of Mo in the $4+$ and $6+$ states suggests that the Mo in the $6+$ state is potentially present as $\mathrm{MoO}_{3}$ and that the $\mathrm{S}$ is bound to $\mathrm{Mo}$ in a mix of $4+$ and $6+$ states, i.e., $\mathrm{MoSO}_{4}$ and $\mathrm{Mo}_{2}\left(\mathrm{SO}_{4}\right)_{3}$, which may both be present on the $\mathrm{MoS}_{2} / \mathrm{C}$ samples. It is interesting to note that 
different sputtering times result in different chemical compositions of $\mathrm{MoS}_{2}$ upon the nanocarbon surface. Note that it has been shown in a study by Latiff et al. ${ }^{31}$ that $\mathrm{MoO}_{\mathrm{x}} \mathrm{compound}$ are not effective catalysts of the HER. However, it is clear that the surface for the most beneficial HER activity corresponds to $\mathrm{MoS}_{2}$ (see Table 1) from sputtering for $45 \mathrm{~min}$, which has the optimal composition. Our observation agrees with independent reports in terms of electrocatalyst composition related to HER performance. ${ }^{32}$

Previous work by Rowley-Neale et al. ${ }^{22}$ has demonstrated that it is possible to incorporate a nanomaterial into the bulk ink of an SPE to fabricate mass-producible and electrocatalytic SPEs, which have scales of economy and are consequently of very low cost. Utilizing the SPE fabrication technique described in that study, summarized in the Experimental Section, the optimized 45 min $\mathrm{MoS}_{2} / \mathrm{C}$ variant was incorporated into the bulk ink of SPEs at a percentage of $2.5,5.0,7.5$, and $10.0 \%$ $\mathrm{MoS}_{2} / \mathrm{C}$ (see Experimental Section), these are denoted as MoS 2 /C-SPEs. The MoS $/$ C-SPEs were explored toward the HER, the results of which are presented in Table 1 and Figure $2 \mathrm{~A}$. All of the $\mathrm{MOS}_{2}$ /C-SPEs display greater HER activity than bare/unmodified SPEs, with a clear trend of increased HER activity with a larger percentage incorporation of $\mathrm{MoS}_{2} / \mathrm{C}$. Thus, the $10 \% \mathrm{MoS}_{2} / \mathrm{C}-\mathrm{SPE}$ displays the most electrocatalytic behavior toward the HER with an onset potential, current density at -0.75 $\mathrm{V}$ (vs SCE), and Tafel slope values of $-0.45 \mathrm{~V}$ (vs SCE) (see Figure S8), $-1.81 \mathrm{~mA} \mathrm{~cm}-2$, and $45 \mathrm{mV}$ dec-1. The optimal electrocatalytic ability of the $10 \%$ MoS2/C-SPE is likely due to it having the largest mass incorporation of $\mathrm{MoS}_{2} / \mathrm{C}$ and, thus, as the work of Kibsgaard and co-workers ${ }^{33}$ suggests, the greatest availability of electrocatalytic edge sites as well as the most beneficial electrical connection between the electrode, electrocatalyst, and electrolyte, therefore the most improved HER catalysis. The relative percentage standard deviations (relative standard deviation (RSD)) for the HER onset potential and achievable currents at $-0.75 \mathrm{~V}$ for the $10 \% \mathrm{MoS}_{2} / \mathrm{C}$-SPE were found to be 0.7 and $4.6 \%(N=5)$, respectively. These small RSD values attest to the reproducibility of the screenprinting technique and the electrodes herein fabricated.

It was important to assess the signal output stability of the $\mathrm{MoS}_{2} / \mathrm{C}$-SPEs. This was undertaken by cycling a 10\% $\mathrm{MoS}_{2}$ / C-SPE 1000 times between 0 and $-1.5 \mathrm{~V}$ (vs SCE) at $100 \mathrm{mV} \mathrm{s}^{-1}$ and holding the potential at $-0.75 \mathrm{~V}$ (vs SCE) for $36000 \mathrm{~s}$. In both cases, the current output of the $10 \% \mathrm{MoS}_{2} / \mathrm{C}-\mathrm{SPE}$ was recorded and displayed in Figure 2B. Note that a carbon counter electrode was utilized during experiments to prevent Pt migration onto the working electrode, which would lead to a convolution of the experiment's outcome..$^{34}$ It was observed that there was a gradual increase of $25.9 \%$ in the achievable current from the 1st to the 1000th scan. This is likely to be due to the $0.5 \mathrm{M} \mathrm{H}_{2} \mathrm{SO}_{4}$ electrolyte partially corroding the binding polymers of the graphitic ink, which leads to a greater number of exposed catalytic edge sites capable of $\mathrm{H}+$ binding or that the electrode surface becomes less hydrophobic over the duration of the test and thus has a greater potential for interaction of the MoS2/C with the electrolyte, 22 creating more favorable triple phase boundaries. In the case of the chronoamperometry (see Figure $2 \mathrm{~B}$, inset), there was a relatively steep decrease in current output from 141 to $124 \mu \mathrm{A}$ after $10000 \mathrm{~s}$; following this, there was a steady decrease to $119 \mu \mathrm{A}$ until 36000 s. Maintaining the potential in a Faradaic region, as we have done herein, will result in the constant production of hydrogen via the HER, this constant bubbling will likely cause a mechanical delamination of the catalyst from the electrode's surface resulting in the observed degradation of current signal output.

\section{CONCLUSIONS}

In this paper, we have implemented and optimized a magnetron sputtering technique to coat a nanocarbon support with $\mathrm{MoS}_{2}$, for the first time, and developed an effective and stable HER catalyst $\left(\mathrm{MoS}_{2} / \mathrm{C}\right)$. The optimized sputtering time was found to correspond to $45 \mathrm{~min}$, at which the most beneficial HER catalysis was observed. Through a thorough XPS analysis, it was determined that a 45 min deposition time correlated with the highest levels of $\mathrm{MoS}_{2}$ present on the sample surfaces, 
thus explaining the optimized catalysis. The $45 \mathrm{~min} \mathrm{MoS}_{2} / \mathrm{C}$ was also incorporated into bespoke screen-printable inks at an optimized mass ratio of $10 \% \mathrm{MoS}_{2} / \mathrm{C}$ to $90 \%$ graphitic. The signal output in regard to HER activity of the subsequently fabricated MOS2/C-SPEs was found to be greatly superior to that of a bare/unmodified SPE with a $0.45 \mathrm{~V}$ (VS SCE) decrease in the HER onset potential and a $1.70 \mathrm{~mA} \mathrm{~cm}^{-2}$ increase in the achievable current density (recorded at $-0.75 \mathrm{~V}$ (vs SCE)).

The use of magnetron sputtering in this manner to fabricate the $\mathrm{MoS}_{2} / \mathrm{C}$ enables the mass production of a $\mathrm{MoS}_{2}$-based electrocatalyst on an industrial scale without the requirement for timeconsuming and low-yield traditional exfoliation methods or the subsequent complexities of trying to electrically wire a 2D-nanomateral to an electrode. $\mathrm{MoS}_{2} / \mathrm{CSPEs}$, therefore, offer a promising, costeffective, tailorable, and mass-producible alternative to Pt and Pt containing materials as the cathodic material involved within an electrolyzer's threephase boundary.

\section{EXPERIMENTAL SECTION}

4.1. Chemicals. All chemicals utilized were of analytical grade and were used as received from SigmaAldrich (U.K.)/ Alfa Aesar without any further purification; this includes the carbon nanopowder (Super P conductive carbon black; diameter: $40-45 \mathrm{~nm},>99 \%$ purity (see Figure S9)). ${ }^{35}$ The $\mathrm{MoS}_{2}$ sputtering target ( $>99.5 \%$ purity) bonded to the copper backing plate was purchased from Teer Coatings (Teer Coatings Ltd., Droitwich, U.K.); the geometrical dimensions of the target are $300 \mathrm{~mm}$ $\times 100 \mathrm{~mm} \times 3 \mathrm{~mm}$. All of the solutions herein utilized were prepared with deionized water of resistivity not less than $18.2 \mathrm{M} \Omega \mathrm{cm}$.

4.2. Electrochemical Measurements. All electrochemical measurements described herein were performed using an Ivium CompactStat (Netherlands) potentiostat. The measurements were implemented utilizing a three-electrode system, where a Pt wire and saturated calomel electrode (SCE) acted as the counter and reference electrodes, respectively. The working electrodes used were either bare/unmodified screenprinted electrodes (SPEs), which were subsequently dropcasted (where an aliquot of the liquid suspended catalyst is deposited using a manual pipette) with $\mathrm{MoS}_{2} / \mathrm{C}$ or SPEs that had MoS2/C incorporated into their bulk inks to produce bulk-modified SPEs. The SPEs were produced via screen printing using an appropriate stencil, which resulted in an electrode working area with a diameter and area of $3 \mathrm{~mm}$ and $0.0707 \mathrm{~cm} 2$, respectively. ${ }^{22,32}$ A DEK 248 screenprinting machine (DEK, Weymouth, U.K.) was used to screen print the electrode configuration onto a polyester (Autostat, $250 \mu \mathrm{m}$ thickness) flexible film after which this layer was cured in a fan oven at $60{ }^{\circ} \mathrm{C} / 30$ min. A dielectric paste (product code D2070423D5; Gwent Electronic Materials Ltd., U.K.) was then utilized to insulate the working electrode area of the printed design from its electrical connection. After curing at $60^{\circ} \mathrm{C} / 30 \mathrm{~min}$, the screen-printed electrodes/surfaces were ready to be used and were connected via an edge connector to ensure a secure electrical connection. ${ }^{36}$

To fabricate the $\mathrm{MoS}_{2} / \mathrm{C}$-SPEs, the $\mathrm{MoS}_{2} / \mathrm{C}$ was incorporated into a pre-existing carbon-graphite ink formulation (product code C2000802P2; Gwent Electronic Materials Ltd., U.K.). Prior to this study, a novel $\mathrm{MoS}_{2}$ ink was considered using a range of solvents, binders, and graphitic materials; however, it was then shown that the produced SPEs had poor/nonexistent electrochemical responses in regard to the HER and oxygen reduction reaction (ORR). ${ }^{32}$ The $\mathrm{MoS}_{2} / \mathrm{C}$ was, therefore, incorporated into the commercially purchased ink on the basis of the mass of particulate (MP) to the mass of the graphitic ink $(\mathrm{MI})$ giving \% = $(\mathrm{MP} / \mathrm{MI}) \times 10$; this approach allows for an effective way to electrically wire/connect the nanomaterial while facilitating the production of mass-producible and reproducible electrocatalytic electrode architectures. The maximum \% incorporation of $\mathrm{MoS}_{2} / \mathrm{C}$ into the ink formula was found to correspond to $40 \%$, as percentage incorporations over this amount resulted in an increase in the viscosity of the ink to such an extent that it was no longer printable via the screen-printing technique herein utilized. It is possible to obtain an estimation as to the total weight incorporation of MoS2/C into each individual SPE by weighing the substrate pre- and postprinting and taking into account the percentage of incorporation. Using this method, it was 
determined that an average weight of 63.4, 126.4, 252.8, and 505.6 $\mu \mathrm{g}$ of $\mathrm{MoS}_{2} / \mathrm{C}$ was incorporated into the 2.5, 5.0, 7.5, and 10.0\% $\mathrm{MoS}_{2} / \mathrm{CSPEs}$. This is, however, an estimation and we, therefore, utilize a percentage denotation throughout this study. The fabricated $\mathrm{MoS}_{2} / \mathrm{C}$ graphitic inks were screen-printed onto the carbon layer of a SPE and subsequently cured for $30 \mathrm{~min}$ at $60^{\circ} \mathrm{C}$ after which they were ready to be tested. For comparative purposes, a platinum polycrystalline electrode (1.6 mm, BAS) was tested as a working electrode toward the HER in the same conditions as the fabricated MoS2/C-SPEs and bare/ unmodified SPEs, to allow for a direct comparison.

The HER measurements were carried out in $0.5 \mathrm{M} \mathrm{H}_{2} \mathrm{SO}_{4}$ with the sulfuric acid solution used being of the highest possible grade available from Sigma-Aldrich (99.999\%, double distilled for trace metal analysis). The $0.5 \mathrm{M} \mathrm{H} 2 \mathrm{SO} 4$ solutions used in the HER was thoroughly deoxygenated via the vigorous bubbling of pure nitrogen through the solution, as is common within the literature. $5 \mathrm{It}$ was essential to remove all traces of oxygen within the test solution, as any oxygen present could be reduced via the oxygen reduction reaction, which could possibly cause an alteration in the observed signal output, leading to a convolution within the results. It should be noted that the HER was determined to start at the potential when the current density deviates from the background current density by $25 \mu \mathrm{A} \mathrm{cm}{ }^{-2}$, as is common within the literature. ${ }^{5,22}$

4.3. Physicochemical Characterization. To perform a thorough and independent physicochemical characterization, the bespoke fabricated $\mathrm{MoS}_{2} / \mathrm{C}$ was analyzed by Raman spectroscopy, scanning electron microscopy (SEM), transmission electron microscopy (TEM), X-ray diffraction (XRD), and Xray photoelectron spectroscopy (XPS). For a full description of the equipment specifications, interested readers are directed to the Supporting Information.

4.4. Fabrication of Bespoke $\mathrm{MoS}_{2}$-Coated Carbon Nanoparticles via Magnetron Sputtering. $\mathrm{MoS}_{2}$ coatings were fabricated in a single-stage process using an arrangement similar to the one reported by Ratova et al., ${ }^{18,19}$ in which particulates, in this case carbon nanoparticles, are manipulated under the magnetrons in an oscillating bowl. In brief, the vacuum coating system used included two planar $300 \mathrm{~mm} \times 100 \mathrm{~mm}$ type II unbalanced magnetrons installed through the top of the chamber in the closed-field configuration facing the oscillator bowl. The $\mathrm{MoS}_{2}$ target was fitted to one of the magnetrons, whereas the other magnetron was covered with the blanking plate and used for closing the magnetic field lines between the magnetrons. The target was sputtered in argon at $15 \mathrm{sccm}$, and the flow of the sputtering gas was regulated using a mass-flow controller. A $5 \mathrm{~kW}$ Advanced Energy Pinnacle Plus magnetron driver was used to power the magnetron; the sputtering was performed in pulsed direct current mode at a time-averaged power of $500 \mathrm{~W}$, a pulse frequency of $100 \mathrm{kHz}$, and duty cycle of $50 \%$. The exact specifics regarding the oscillating bowl can be found in the Supporting Information. Each deposition cycle consisted of a $5 \mathrm{~g}$ charge of carbon nanopowder, which was loaded into the oscillator bowl, the chamber was then evacuated to a base pressure of lower than 1 $\times 10^{-3} \mathrm{~Pa}$. Sputtering times of $7.5,15,30,45,60$, and 120 min were used to vary the $\mathrm{MoS}_{2}$ loading on the samples. Note that this is an unlikely translation to other systems and diligent time-controlled experiments will need to be undertaken. A summary of the $\mathrm{MoS}_{2}$ sputtering conditions is presented in Table 3. 
Table 3. Summary of Magnetron Sputtering Deposition Conditions for the Fabrication of the MoS2/C Samples

$\begin{array}{ll}\text { deposition parameters } & \\ \text { target material } & \mathrm{MoS}_{2} \\ \text { substrate material } & \text { carbon nanopowder }(5 \mathrm{~g}) \\ \text { Ar flow } & 15 \mathrm{sccm} \\ \text { process pressure } & 2 \times 10^{-1} \mathrm{~Pa} \\ \text { deposition time } & 7.5,15,30,45,60,120 \mathrm{~min} \\ \text { time-average power } & 500 \mathrm{~W} \\ \text { target current } & 0.89 \mathrm{~A} \\ \text { target voltage } & -560 \mathrm{~V}\end{array}$

\section{ASSOCIATED CONTENT}

\section{* Supporting Information}

The Supporting Information is available free of charge on the ACS Publications website at DOI: 10.1021/acsomega.8b00258. Physicochemical characterization instrumentation, oscillating bed mechanism, XPS spectra and analysis, EDX analysis, Tafel analysis, SEM and TEM images, Raman spectra, XRD (PDF)

\section{AUTHOR INFORMATION}

\section{Corresponding Author}

*E-mail: c.banks@mmu.ac.uk.Tel: ++(0)1612471196. Fax: +

$+(0) 1612476831$. Website: www.craigbanksresearch.com.

ORCID

Craig E. Banks: 0000-0002-0756-9764

Notes

The authors declare no competing financial interest.

\section{ACKNOWLEDGMENTS}

Funding from the Engineering and Physical Sciences Research Council (Reference: EP/N001877/1), British Council Institutional Grant Link (No. 172726574) is acknowledged. The Manchester Fuel Cell Innovation Centre is funded by the European Regional Development Fund. 


\section{REFERENCES}

(1) Schultz, M. G.; Diehl, T.; Brasseur, G. P.; Zittel, W. Air Pollution and Climate-Forcing Impacts of a Global Hydrogen Economy. Science 2003, 302, 624-627.

(2) Ahmed, A.; Al-Amin, A. Q.; Ambrose, A. F.; Saidur, R. Hydrogen Fuel and Transport System: A Sustainable and Environmental Future. Int. J. Hydrogen Energy 2016, 41, 1369-1380.

(3) Ji, S.; Yang, Z.; Zhang, C.; Liu, Z.; Tjiu, W. W.; Phang, I. Y.; Zhang, Z.; Pan, J.; Liu, T. Exfoliated MoS2 Nanosheets as Efficient Catalysts for Electrochemical Hydrogen Evolution. Electrochim. Acta 2013, 109, 269-275.

(4) Lei, Z.; Xu, S.; Wu, P. Ultra-Thin and Porous MoSe2 Nanosheets: Facile Preparation and Enhanced Electrocatalytic Activity Towards the Hydrogen Evolution Reaction. Phys. Chem. Chem. Phys. 2016, $18,70-74$.

(5) Rowley-Neale, S. J.; Brownson, D. A. C.; Smith, G. C.; Sawtell, D. A. G.; Kelly, P. J.; Banks, C. E. 2D Nanosheet Molybdenum Disulphide (MoS2) Modified Electrodes Explored Towards the Hydrogen Evolution Reaction. Nanoscale 2015, 7, 18152-18168.

(6) Voiry, D.; Salehi, M.; Silva, R.; Fujita, T.; Chen, M.; Asefa, T.; Shenoy, V. B.; Eda, G.; Chhowalla, M. Conducting MoS2 Nanosheets as Catalysts for Hydrogen Evolution Reaction. Nano Lett. 2013, 13, 6222-6227.

(7) Lazar, P.; Otyepka, M. Role of the Edge Properties in the Hydrogen Evolution Reaction on MoS2. Chem. - Eur. J. 2017, 23, 4863-4869.

(8) Li, G.; Zhang, D.; Qiao, Q.; Yu, Y.; Peterson, D.; Zafar, A.; Kumar, R.; Curtarolo, S.; Hunte, F.; Shannon, S.; Zhu, Y.; Yang, W.; Cao, L. All The Catalytic Active Sites of MoS2 for Hydrogen Evolution. J. Am. Chem. Soc. 2016, 138, 16632-16638.

(9) Chia, X.; Eng, A. Y. S.; Ambrosi, A.; Tan, S. M.; Pumera, M. Electrochemistry of Nanostructured Layered Transition-Metal Dichalcogenides. Chem. Rev. 2015, 115, 11941-11966.

(10) Franceschini, E. A.; Lacconi, G. I.; Corti, H. R. Kinetics of the hydrogen evolution on nickel in alkaline solution: new insight from rotating disk electrode and impedance spectroscopy analysis. Electrochim. Acta 2015, 159, 210-218.

(11) Hinnemann, B.; Moses, P. G.; Bonde, J.; Jørgensen, K. P.; Nielsen, J. H.; Horch, S.; Chorkendorff, I.; Nørskov, J. K. Biomimetic Hydrogen Evolution: MoS2 Nanoparticles as Catalyst for Hydrogen Evolution. J. Am. Chem. Soc. 2005, 127, 5308-5309.

(12) Toh, R. J.; Sofer, Z.; Luxa, J.; Pumera, M. Ultrapure Molybdenum Disulfide Shows Enhanced Catalysis for Hydrogen Evolution over Impurities-Doped Counterpart. ChemCatChem 2017, 9, 1168-1171.

(13) Mun, J.; Kim, D.; Yun, J.; Shin, Y.; Kang, S.; Kin, T. Chemical Vapor Deposition of MoS2 Films. ESC Trans. 2013, 58, 199-202.

(14) Niu, L.; Coleman, J. N.; Zhang, H.; Shin, H.; Chhowalla, M.; Zheng, Z. Production of TwoDimensional Nanomaterials via Liquid- Based Direct Exfoliation. Small 2016, 12, 272-293.

(15) Li, H.; Wu, J.; Yin, Z.; Zhang, H. Preparation and Applications of Mechanically Exfoliated SingleLayer and Multilayer MoS2 and WSe2 Nanosheets. Acc. Chem. Res. 2014, 47, 1067-1075.

(16) Varrla, E.; Backes, C.; Paton, K. R.; Harvey, A.; Gholamvand, Z.; McCauley, J.; Coleman, J. N. Large-Scale Production of Size- Controlled MoS2 Nanosheets by Shear Exfoliation. Chem. Mater. 2015, 27, 1129-1139.

(17) Kelly, P. J.; Arnell, R. D. Magnetron sputtering: a review of recent developments and applications. Vacuum 2000, 56, 159-172. 
(18) Ratova, M.; Kelly, P.; West, G.; Tosheva, L. A Novel Technique for the Deposition of Bismuth Tungstate onto Titania Nanoparticulates for Enhancing the Visible Light Photocatalytic Activity. Coatings 2016, 6, 29.

(19) Ratova, M.; Kelly, P. J.; West, G. T.; Tosheva, L.; Edge, M. Reactive magnetron sputtering deposition of bismuth tungstate onto titania nanoparticles for enhancing visible light photocatalytic activity. Appl. Surf. Sci. 2017, 392, 590-597.

(20) Escalera-López, D.; Niu, Y.; Yin, J.; Cooke, K.; Rees, N. V.; Palmer, R. E. Enhancement of the Hydrogen Evolution Reaction from Ni-MoS2 Hybrid Nanoclusters. ACS Catal. 2016, 6, 6008-6017.

(21) Abbaspour, A.; Mirahmadi, E. Electrocatalytic hydrogen evolution reaction on carbon paste electrode modified with Ni ferrite nanoparticles. Fuel 2013, 104, 575-582.

(22) Rowley-Neale, S. J.; Foster, C. W.; Smith, G. C.; Brownson, D. A. C.; Banks, C. E. Mass-producible 2D-MoSe2 bulk modified screenprinted electrodes provide significant electrocatalytic performances towards the hydrogen evolution reaction. Sustainable Energy Fuels 2017, 1, 74-83.

(23) Wang, H.; Kong, D.; Johanes, P.; Cha, J. J.; Zheng, G.; Yan, K.; Liu, N.; Cui, Y. MoSe2 and WSe2 Nanofilms with Vertically Aligned Molecular Layers on Curved and Rough Surfaces. Nano Lett. 2013, 13, 3426-3433.

(24) Benck, J. D.; Chen, Z.; Kuritzky, L. Y.; Forman, A. J.; Jaramillo, T. F. Amorphous Molybdenum Sulfide Catalysts for Electrochemical Hydrogen Production: Insights into the Origin of their Catalytic Activity. ACS Catal. 2012, 2, 1916-1923.

(25) Zhu, Q.; Zhao, C.; Bian, Y.; Mao, C.; Peng, H.; Li, G.; Chen, K. MoS2/nitrogen-doped carbon hybrid nanorods with expanded interlayer spacing as an advanced anode material for lithium ion batteries. Synth. Met. 2018, 235, 103-109.

(26) Li, H.; Zhang, Q.; Yap, C. C. R.; Tay, B. K.; Edwin, T. H. T.; Olivier, A.; Baillargeat, D. From Bulk to Monolayer MoS2: Evolution of Raman Scattering. Adv. Funct. Mater. 2012, 22, 1385-1390.

(27) Naebe, M.; Wang, J.; Amini, A.; Khayyam, H.; Hameed, N.; Li, L. H.; Chen, Y.; Fox, B. Mechanical Property and Structure of Covalent Functionalised Graphene/Epoxy Nanocomposites. Sci. Rep. 2014, 4, No. 4375.

(28) Kumar, M. P.; Kesavan, T.; Kalita, G.; Ragupathy, P.; Narayanan, T. N.; Pattanayak, D. K. On the large capacitance of

nitrogen doped graphene derived by a facile route. RSC Adv. 2014, 4, 38689-38697.

(29) Joensen, P.; Crozier, E. D.; Alberding, N.; Frindt, R. F. A Study of Single-Layer and Restacked MoS2 by X-ray Diffraction and X-ray Absorption Spectroscopy. J. Phys. C: Solid State Phys. 1987, 20, 4043-4053.

(30) Shi, Y.; Zhou, W.; Lu, A.-Y.; Fang, W.; Lee, Y.-H.; Hsu, A. L.; Kim, S. M.; Kim, K. K.; Yang, H. Y.; Li, L.-J.; Idrobo, J.-C.; Kong, J. van der Waals Epitaxy of MoS2 Layers Using Graphene As Growth Templates. Nano Lett. 2012, 12, 2784-2791.

(31) Latiff, N. M.; Wang, L.; Mayorga-Martinez, C. C.; Sofer, Z.; Fisher, A. C.; Pumera, M. Valence and oxide impurities in MoS2 and WS2 dramatically change their electrocatalytic activity towards proton reduction. Nanoscale 2016, 8, 16752-16760.

(32) Rowley-Neale, S. J.; Smith, G. C.; Banks, C. E. Mass-Producible 2D-MoS2-Impregnated ScreenPrinted Electrodes That Demonstrate Efficient Electrocatalysis toward the Oxygen Reduction Reaction. ACS Appl. Mater. Interfaces 2017, 9, 22539-22548.

(33) Kibsgaard, J.; Chen, Z.; Reinecke, B. N.; Jaramillo, T. F. Engineering the surface structure of MoS2 to preferentially expose active edge sites for electrocatalysis. Nat. Mater. 2012, 11, 963. 
(34) Gottlieb, E.; Kopeć, M.; Banerjee, M.; Mohin, J.; Yaron, D.; Matyjaszewski, K.; Kowalewski, T. InSitu Platinum Deposition on Nitrogen-Doped Carbon Films as a Source of Catalytic Activity in a Hydrogen Evolution Reaction. ACS Appl. Mater. Interfaces 2016, 8, 21531-21538.

(35) Aesar, A. https://www.alfa.com/en/catalog/H30253/ (accessed Dec 18, 2017).

(36) Galdino, F. E.; Foster, C. W.; Bonacin, J. A.; Banks, C. E. Exploring the Electrical Wiring of ScreenPrinted Configurations Utilised in Electroanalysis. Anal. Methods 2015, 7, 1208-1214. 